\title{
The Relative Neighbourhood Graph is a part of every $30^{\circ}$-Triangulation
}

\author{
J. Mark Keil*
}

\section{Abstract}

We study sets of points in the two-dimensional Euclidean plane. The relative neighbourhood graph (RNG) of a point set is a straight line graph that connects two points from the point set if and only if there is no other point in the set that is closer to both points than they are to each other. A triangulation of a point set is a maximal set of nonintersecting line segments (called edges) with vertices in the point set. We introduce angular rectrictions in the triangulations. Using the well-known method of exclusion regions, we show that the relative neighbourhood graph is a part of every triangulation all of the angles of which are greater than or equal to $30^{\circ}$.

Keywords: triangulation, relative neighbourhood graph, angular restriction, exclusion region

\section{Introduction and basic definitions}

Definition 1 For a given planar set of points $S$, the relative neighbourhood graph of $S$, denoted by $R N G(S)$ consists of all edges $A B$, where $A, B \in S$, such that there is no point from $S$ that is closer to both $A$ and $B$ than the distance $A B$.

This definition is equivalent to saying that the region formed by the intersection of the circles with radii $|A B|$ centered at $A$ and $B$ is empty of points of $S$. This region is known in the literature as a lune of the edge $A B$. The lune is illustrated in Figure 1.

The relative neighbourhood graph has been extensively studied with relation to optimal triangulations. The RNG is a subsgraph of the Delaunay triangulation and the Gabriel graph of a point set, and it is a supergraph of the Minimum Spanning Tree of the point set [1]. The RNG is therefore a connected graph, with linear number of edges in $n$ - the size of the point set $S$. The RNG is a subgraph of the MinMax Length triangulation as shown in [5]. Another interesting result there is that the RNG subdivides the convex hull of the point set into simple polygons, and each of these polygons contains at most one convex hull edge. The connectivity is a very important property of RNG as it automatically implies a polynomial time computability of any optimal triangulation

\footnotetext{
*Department of Computer Science, University of Saskatchewan, keil@cs.usask.ca

$\dagger$ Department of Computer Science, University of Saskatchewan, tsv552@mail.usask.ca
}

\author{
Tzvetalin S. Vassilev ${ }^{\dagger}$
}

of which it is a part. To be more exact, this can be done in $O\left(n^{3}\right)$ time and $O\left(n^{2}\right)$ space by Klincesk's algorithm (dynamic programming) [4].

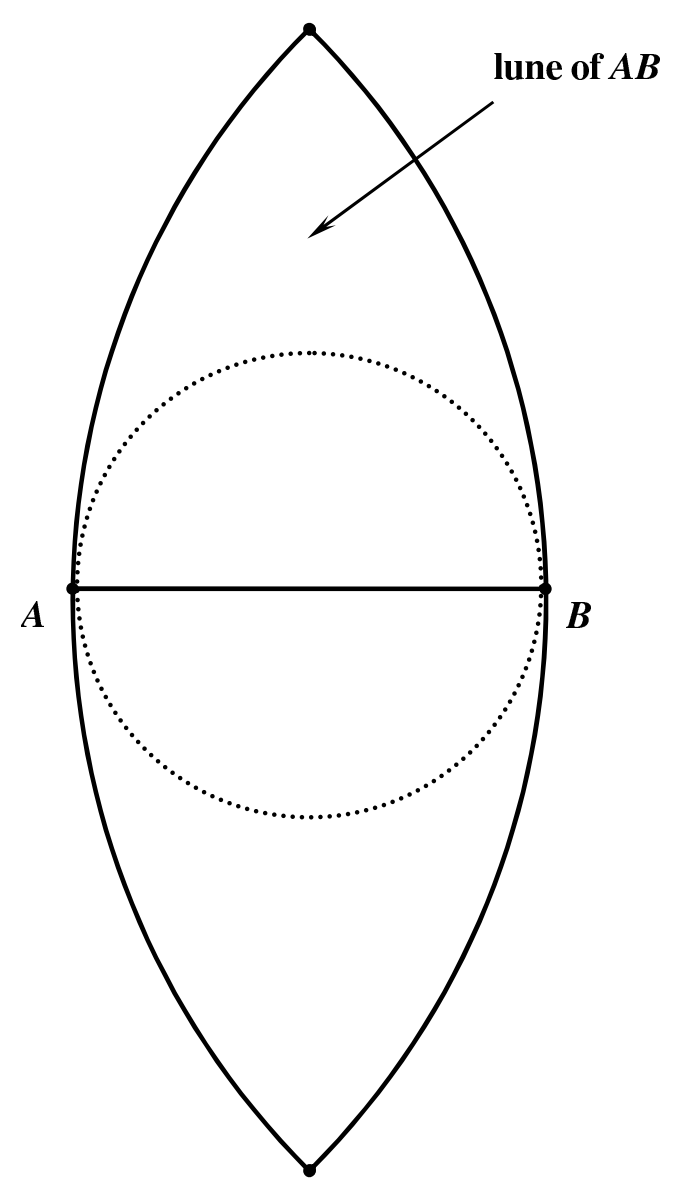

Figure 1: The lune of the edge $A B$

Definition 2 ( $\alpha$-triangulation) Given a planar point set $S$ and an angle $\alpha$ such that $0^{\circ}<\alpha \leq 60^{\circ}$, a triangulation $T$ of $S$ is called $\alpha$-triangulation if and only if all the angles in the triangles of $T$ are greater than or equal to $\alpha$.

Angular constraints are sometimes dicated by the application. In general it is considered that "fat" triangulations, i.e. triangulation with no small angles are more suitable for specific purposes as mesh generation, for example. Interesting experimental results on angle-constrained triangulations are presented in [2]. 
Definition 3 (Exclusion region) Given a planar point set $S$ and two points $A$ and $B$ of $S$, the exclusion region of the edge $A B$ is a closed planar region with the property that its interior is empty of other points of $S$ when the edge $A B$ is a part of a specific (optimal) triangulation.

Thus, the exclusion region is defined with reference to some quality measure or a specific property of the triangulation. The boundary of the exclusion region is usually a chain of line segments, circular arcs, etc. One classical example is the diamond-shaped exclusion region for the Minimum Weight Triangulation [3]. Conversely, we are interested in the property (which originally gave the name) that if the named region for an edge contains point(s) from the point set, the edge can be excluded from consideration as it will not be a part of any triangulation with the desired property. Next we discuss the exclusion region that results from introducing angular constraints, we call it a forbidden zone.

\section{Angular constraints and forbidden zones}

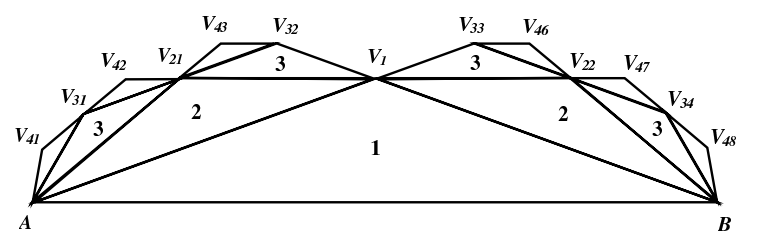

Figure 2: Construction of the $i$-th (for $i \leq 4$ ) order triangles for $\alpha=20^{\circ}$

Definition 4 (Forbidden zone) Given a planar point set $S$ and an angle $\alpha$ such that $0^{\circ}<\alpha \leq 60^{\circ}$ we call an edge $A B, A, B \in S$ internal if there are points from $S$ on both sides of the line $A B$. For an internal edge $A B$ we define the isosceles triangle $\triangle A V_{1} B$ with angles $\angle V_{1} A B=\angle A B V_{1}=\alpha$ as a first order triangle with respect to the edge $A B$. We call the point $V_{1}$ a first order vertex with respect to the edge $A B$. Similarly, we call the edges $A V_{1}$ and $V_{1} B$ first order edges with respect to the edge $A B$. Note that the point $V_{1}$ might not be, and generally is not a point from the set $S$. It is just a part of an auxiliary construction. Recursively, on each $i$-th order edge we can build an isosceles triangle with base angles of $\alpha$ and it will be $(i+1)$-th order triangle with respect to the original edge $A B$. As it is clear from the construction method, for $i>1$ there are multiple $i$-th order triangles, edges and vertices. The vertices in particular can be enumerated by double indexing $V_{i k}$ meaning that $V_{i k}$ is the $k$-th vertex of $i$-th order, where $i=2,3, \ldots, k=1,2, \ldots, 2^{i-1}$. The construction is illustrated in Figure 2. For each triangle of $i$-th order we can define its free wedge as the interior of the angle opposite of its internal angle of $180^{\circ}-2 \alpha$. The union over all values of $i, i=1,2, \ldots$ of all free wedges for all $i$-th order triangles of the edge $A B$ is the free zone of the edge $A B$. Note that some of the $i$-th order triangles lie entirely in the free wedges of triangles of lower order, thus not contributing to the free zone. Also some of the wedges overlap. The complement of the free zone of the edge $A B$ is the forbidden zone of the edge $A B$.

Lemma 1 If there is a point of the set $S$ in the forbidden zone of the edge $A B$, then this edge is not a part of any $\alpha$-triangulation of $S$.

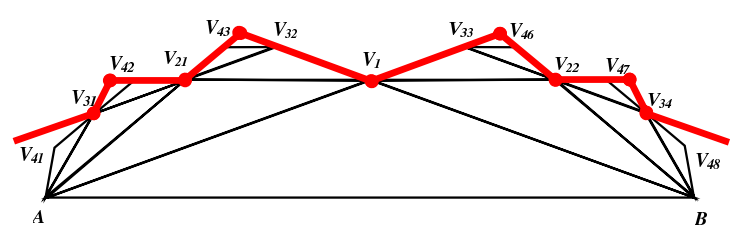

Figure 3: The forbidden zone of the edge $A B$, the boundary line (red) up to $3^{\text {rd }}$ order

Proof. In fact the forbidden zone was built so as to ensure this property. To see that it is valid, consider the location of a point $X \in S$ inside the forbidden zone. Remember that by our assumption the edge $A B$ is in some $\alpha$-triangulation of $S$. By construction $X$ is in the closure of some $i$-th order triangle of the edge $A B$. Suppose $i=1$, i.e. the point $X$ is in the first order triangle $\triangle A V_{1} B$. Then $X$ is either connected directly to the edge $A B$, which forms an illegal triangle (both angles at $A$ and $B$ will be less than $\alpha$ ), or there is another edge of the $\alpha$-triangulation that intersects the interior of the triangle $\triangle A X B$. In the latter case, consider the "closest" to $A B$ edge with this property, one of the points $A$ or $B$ has to be connected to an endpoint of this edge, thus violating the angular constraint. Therefore the first order triangle $\triangle A V_{1} B$ is empty of points of $S$. Let now $i=2$ and assume that $X$ is inside $\triangle V_{1} V_{22} B . X$ cannot be connected directly to $A$ as this will immediately violate the angular constraint. Thus there is an edge intersecting the interior of $\triangle V_{1} V_{22} B$. Similarly to the previous case there will be an edge emanating form $B$ that either connects to $X$ or to an endpoint of the "closest" edge. However the edge $A B$ is connected to some point in the triangulation. Therefore we have two edges emanating from $B$ inside an angle of less than $2 \alpha$, and this violates the angular constraint. Inductively, by considering the edges of some triangulation emanating from the points $A$ and $B$, and their intersection with the interiors of the triangles of order up to $i$ we will be able to show that any triangulation containing the edge $A B$ contains angle(s) smaller than 
$\alpha$ which shows the validity of the argument.

In Figure 3 the boundary line of the forbidden zone is shown in red (bold). The forbidden zone extends on both sides of the edge $A B$, although in the construction we implicitly referred only to one of the halfplanes defined by the line $A B$.

Corollary 2 Suppose that the edge $A B$ has length $2 a$, and that $\alpha=30^{\circ}$. The forbidden zone of the edge $A B$ includes a rectangle with base $A B$ and height $\frac{a}{\sqrt{3}}$. On top of this rectangle the forbidden zone includes two right triangles $\triangle V_{21} V_{L} V_{1}$ and $\triangle V_{1} V_{R} V_{22}$ with bases of $\frac{2 a}{3}$ and angles of $30^{\circ}$ at $V_{1}$. Refer to Figure 4.

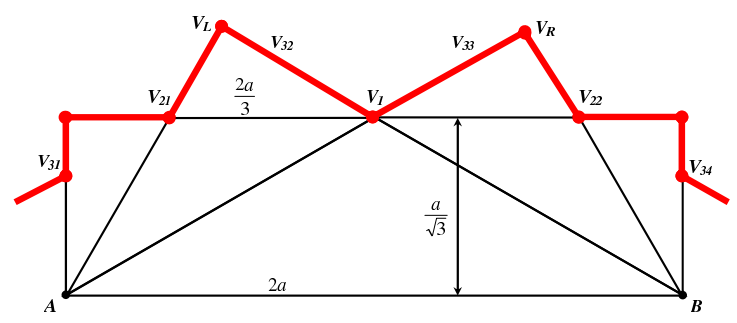

Figure 4: Parameters of the forbidden zone of the edge $A B=2 a$ for $\alpha=30^{\circ}$

\section{Main result}

We are going to show that the RNG is a part of every $30^{\circ}$-triangulation by showing that the forbidden zones of all possible edges that could intersect a given edge $A B$ of the RNG contain at least one of the points $A$ or $B$ in their interior. Thus, in an $30^{\circ}-$ triangulation, if such exists, the RNG edges cannot be intersected by other legal edges.

Keeping the assumptions of the previous section, let us denote the midpoint of the considered edge $A B$ by $O$. Further, let us place the edge $A B$ on the $x$-axis of a coordinate system with an origin in its midpoint $O$. The points $A$ and $B$ have coordinates $(-a, 0)$ and $(a, 0)$, respectively, where $a$ is a positive real number.

Lemma 3 Let $P Q$ be a segment that goes through $O$ and the points $P$ and $Q$ are on the boundary of the lune of $A B$. Then the point $B$ is in the forbidden zone of the segment $P Q$.

Proof. Without loss of generality we can assume that the point $P$ lies in the I quadrant. Let $P(x, y)$, denote the orthogonal projection of the point $B$ onto the segment $P Q$ by $H_{B}$. The idea is to compute the distance $B H_{B}$ and the depth of the forbidden zone of $P Q$ at $H_{B}$ and show that the first is less than or equal to the second quantity, thus establishing the claim. It is easy to see that $H_{B}$ is a point that lies in the segment $O P$ : the angles of triangle $\triangle O B P$ are all acute and $B H_{B}$ is an altitude in this triangle. Using the fact that $P$ lies on a circle centered at $A$ with a radius of $2 a$, and the law of sines we obtain $O H_{B}=\sqrt{\frac{a}{3 a-2 x}} \cdot x$ and $B H_{B}=\sqrt{\frac{a(a-x)(3 a+x)}{3 a-2 x}}$. Denote the distance between $O$ and $P$ by $p$, and the distance between $O$ and $H_{B}$ by $d$. We can write the depth of the forbidden zone $B(d)$ as a function of $d$ and $p$ as follows:

$$
B(d)= \begin{cases}(p+d) / \sqrt{3} & \text { for } 0 \leq d \leq p / 2 \\ \sqrt{3}(p-d) & \text { for } p / 2 \leq d \leq 2 p / 3 \\ p / \sqrt{3} & \text { for } 2 p / 3 \leq d \leq p\end{cases}
$$

We have to verify, therefore, that $B H_{B} \leq B(d)$ for all values of $x$ and $a$. This is rather lengthy, but only involves basic mathematics and is omitted here. The result of this lemma was suggested by computational experiments done by the first author using the software package Cinderella (C).

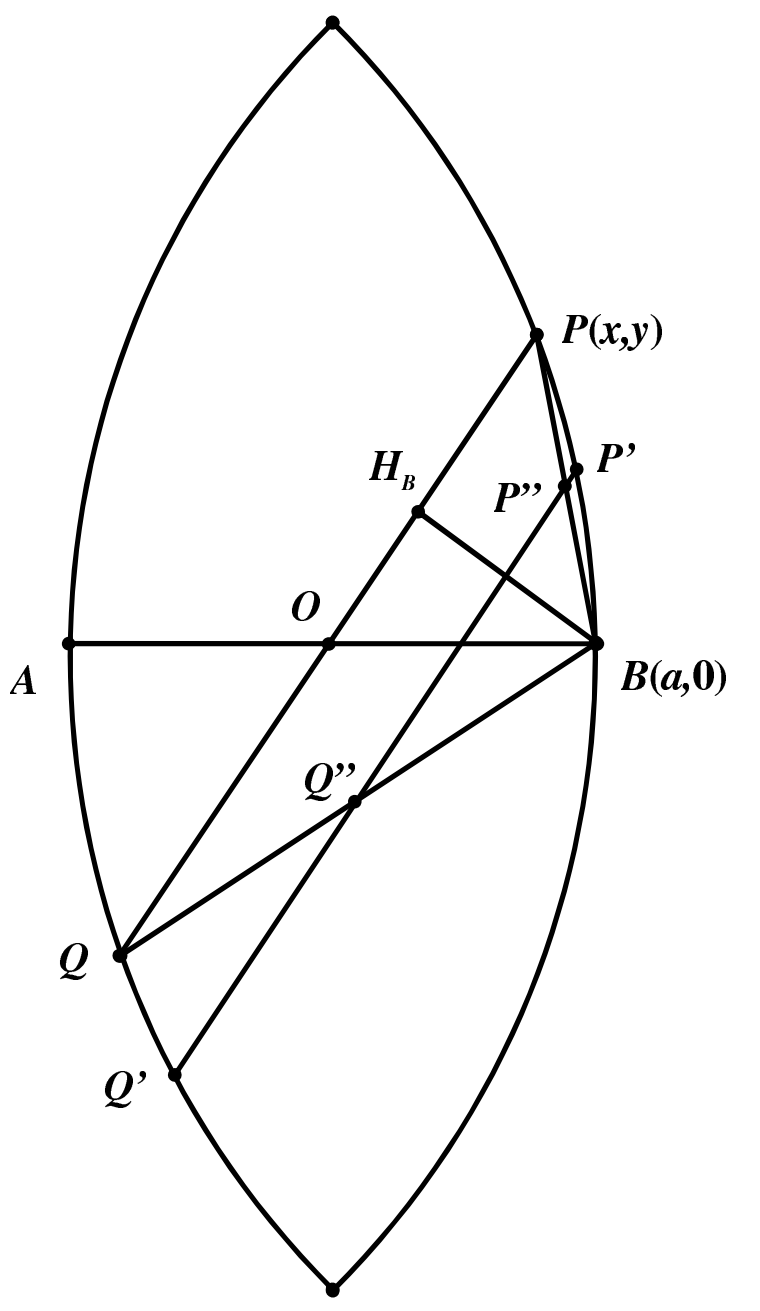

Figure 5: Edges $P Q$ and $P^{\prime} Q^{\prime}$ intersecting the RNG edge $A B$ in Lemmas 3 and 5 
Corollary 4 If a segment $P^{\prime} Q^{\prime}$ properly contains another segment $P^{\prime \prime} Q^{\prime \prime}$ then the forbidden zone of $P^{\prime} Q^{\prime}$ properly contains the forbidden zone of $P^{\prime \prime} Q^{\prime \prime}$.

Proof. From the analytical geometry approach taken in the proof of Lemma 3, we can use Equation 1 which is valid in general, i.e. it describes the (left) part of the forbidden zone of a segment of length $2 p$ with respect to a point that is at a distance $d$ from the midpoint of that segment. Now, consider extension of the segment to the left by a length of $2 h$. In analytical form it corresponds to the substitution:

$$
\{p \leftarrow p-h, d \leftarrow d-h\}
$$

It is now easily verified by Equation 1 that each point that was in the forbidden zone of the original segment is also in the forbidden zone of the extended segment. Similarly, an extension of the segment to the right by a length of $2 h$ is equivalent to the substitution:

$$
\{p \leftarrow p+h, d \leftarrow d+h\}
$$

Again, Equation 1 verifies that any point that was in the forbidden zone of the segment before its extension is still in the forbidden zone after the extension. Figure 6 presents an illustration.

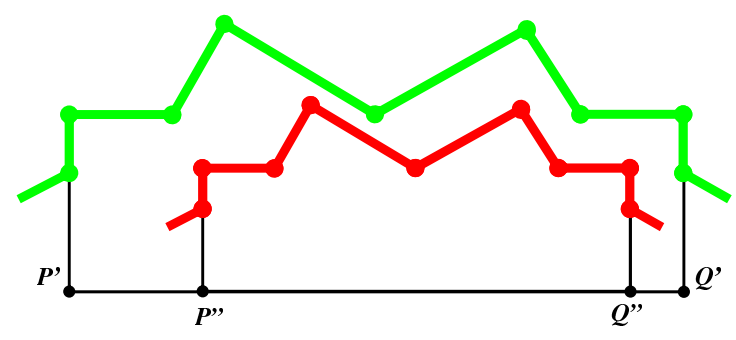

Figure 6: Edges $P^{\prime} Q^{\prime}$ and $P^{\prime \prime} Q^{\prime \prime}$ and their respective forbidden zone boundaries

Further, we have to consider possible edges that cross $A B$ outside of its midpoint.

Lemma 5 Let $P^{\prime} Q^{\prime}$ be a segment such that the points $P^{\prime}$ and $Q^{\prime}$ are on the boundary of the lune of $A B$. Let $P^{\prime} Q^{\prime}$ intersect $A B$ at a point $R$ such that $R$ is between $O$ and $B$. Then the point $B$ is in the forbidden zone of the segment $P^{\prime} Q^{\prime}$.

Proof. As in the proof of Lemma 3, assume that the point $P^{\prime}$ is in the I quadrant, further assume that the edge $P Q$ through $O$ is parallel to the edge $P^{\prime} Q^{\prime}$. The situation is illustrated in Figure 5. Construct the segment $B P$, by construction and the properties of the lune/circles, $B P$ intersects $P^{\prime} Q^{\prime}$ in an internal point, which we denote by $P^{\prime \prime}$. Similarly, if we construct the segment $B Q$ it will intersect $P^{\prime} Q^{\prime}$ at an internal point which we denote by $Q^{\prime \prime}$. By construction $\triangle P B Q \sim \triangle P^{\prime \prime} B Q^{\prime \prime}$ because of the fact that $P Q$ and $P^{\prime \prime} Q^{\prime \prime}$ are parallel. Because of the similarity of the two triangles and the scaling property the fact that $B$ is in the forbidden zone of $P Q$, established in Lemma 3 , implies that $B$ is also in the forbidden zone of $P^{\prime \prime} Q^{\prime \prime}$. Thus, we have two segments, namely $P^{\prime \prime} Q^{\prime \prime}$ and $P^{\prime} Q^{\prime}$ that satisfy the premises of Corollary 4. We conclude that $B$ is in the forbidden zone of the edge $P^{\prime} Q^{\prime}$. Since any edge crossing $A B$ is parallel to an edge crossing $A B$ and going through its midpoint, the claim of this lemma is established.

Theorem 6 The relative neighbourhood graph of a planar set of points is part of every $30^{\circ}$-triangulation of this set (if such a triangulation exists).

Proof. It is evident form Lemmas 3 and 5 that the edges of the relative neighbourhood graph of a planar point set $S$ cannot be intersected by any other edge in a $30^{\circ}$-triangulation, if such a triangulation exists. Therefore, they must be in every $30^{\circ}$-triangulation, if such a triangulation exists.

\section{Conclusion}

The result presented in this paper is tight. In other words, there is no guarantee that for an angle $\alpha<30^{\circ}$ the relative neighbourhood graph will be part of every (or any) $\alpha$-triangulation. A four-point example can be constructed that shows this. Consider a pair of points $A$ and $B$ as per the notation used throughout this paper, and two other points $C$ and $D$ "slightly" outside the lune of $A B$, placed on the right side of the perpendicular bisector of $A B$ infinitesimally close to it. Analysis shows that we can make the angles $\angle D C B$ and $\angle C D B$ as close to $30^{\circ}$ as we want, while keeping the point $B$ outside of the forbidden zone of the edge $C D$.

\section{References}

[1] M. de Berg, M. van Kreveld, M. Overmars, and O. Schwarzkopf. Computational Geometry: Algorithms and Applications, 2nd Edition. SpringerVerlag, February 2000.

[2] Y. Dai, N. Katoh, and S.-W. Cheng. LMT-skeleton Heuristics for Several New Classes of Optimal Triangulations. Computational Geometry: Theory and Applications. Vol. 17, 2000, pages 51-68.

[3] R. L. Drysdale, S. McElfresh, and J. S. Snoeyink. On Exclusion regions for Optimal Triangulations. Discrete Applied Mathematics. Vol. 109, 2001, pages 4965.

[4] G. T. Klincsek. Minimal Triangulations of Polygonal Domains. Annals of Discrete Mathematics. Vol. 9, 1980, pages 121-123.

[5] T. S. Tan. Optimal Two-dimensional Triangulations. Ph.D. Thesis, University of Illinois at UrbanaChampaign, Urbana, 1993. 